\title{
Analysis of Asymmetrical Modes in Medium Voltage Electrical Grids with Compensated Neutral
}

\author{
Stanislav Girshin, Vladimir Goryunov, Evgenii Kuznetsov, Dmitrii Safonov and Elena Petrova \\ Omsk State Technical University, Energy Department, 644050 pr. Mira 11, Omsk, Russian Federation
}

\begin{abstract}
A mathematical model of steady-state radial medium voltage electrical network with earthed neutral via arc-suppression coils has been carried out. A detailed equivalent circuit of the examined network using the completed equivalent circuit of phase coordinates methods and Kirchhoff's laws has been developed. The system of mathematical models equations has been completed. The advantage of the created mathematical model is the ability to increase the accuracy of the simulation by a detailed account of features of a network with arc-suppression coils. The mathematical model considers the load currents, the non-linear nature of consumers, the impact of the power lines asymmetry. Using the developed mathematical model there are presented results of the carried out researches of three power lines with different cross sections with neutral grounding via arc-suppression coils. The analysis of asymmetry parameters impact of power lines on the value of the neutral displacement voltage and on the voltage value at the end of the lines at different values of the reactor inductance has been completed. The conclusion about the model possibility to perform validation of the reactor tuning, to evaluate voltage quality parameters, to carry out the formation of reliable initial conditions for calculating the overload in the single-phase earth faults. In conclusion the model performs validation of the reactor tuning and it evaluates voltage quality parameters in order to carry out the formation of reliable initial conditions. It also calculates the overload in the single-phase earth faults.
\end{abstract}

\section{Introduction}

Electrical medium voltage networks of a large number of countries (Germany, Austria, France, Italy, Finland, Sweden, Norway, Poland, Czech Republic, Russia, and others) use the mode of neutral grounding via arcsuppression coils. The popularity of this mode is due to the benefits of eliminating negative results common for electrical networks of single-phase earth fault. When single-phase ground fault the arc-suppression coils creates at the point of fault inductive current equal to the capacitative current. As a result, the total current of the fault is virtually zero, so there is no need for a fast tripping of the damaged power lines. The effectiveness of arc-suppression coils when faults in the network can be improved with further development of automated control systems of arc-suppression coils. This task can be achieved when using mathematical models of the electrical network with arc-suppression coils adequately reflecting the processes. One of the important features of the medium voltage electrical networks is asymmetry of operating modes, due to the presence of sufficiently long and untransposed lines.

Especially important asymmetry is in networks with compensation of capacitative currents and for solving problems connected with operation modes of neutral [1][4]. The presence of natural asymmetry can lead to abnormally large voltage of neutral displacement in normal modes [5]-[8] and requires detailed consideration. Moreover, the mode asymmetry leads to increased energy losses in the power network compared to a symmetric mode in the same consumer loads. Therefore, it is of interest to analyze such modes from energy efficiency points. Different approaches to the calculation of electric networks modes are presented in [9], [10]. Carried out and presented in this paper researches are to improve the accuracy of asymmetrical modes simulation considering not only the cross but also longitudinal equivalent circuits of the power line, as well as other characteristics of electrical networks with compensated neutral. The advanced mathematical model which allows to improve the quality of the research has been developed. This approach makes it possible to find the actual voltage drop in the examined electrical networks, the real voltage distribution considering asymmetry and determine the magnitude of the neutral displacement.

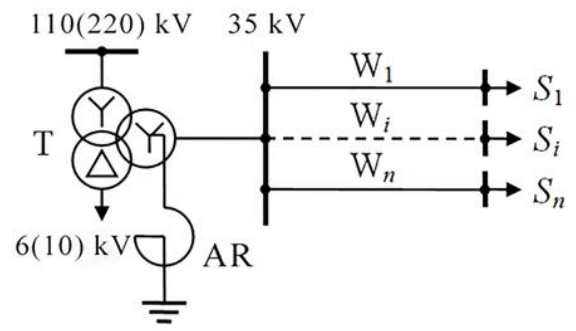

Figure 1. $35 \mathrm{kV}$ radial grid with arc-suppression coils 


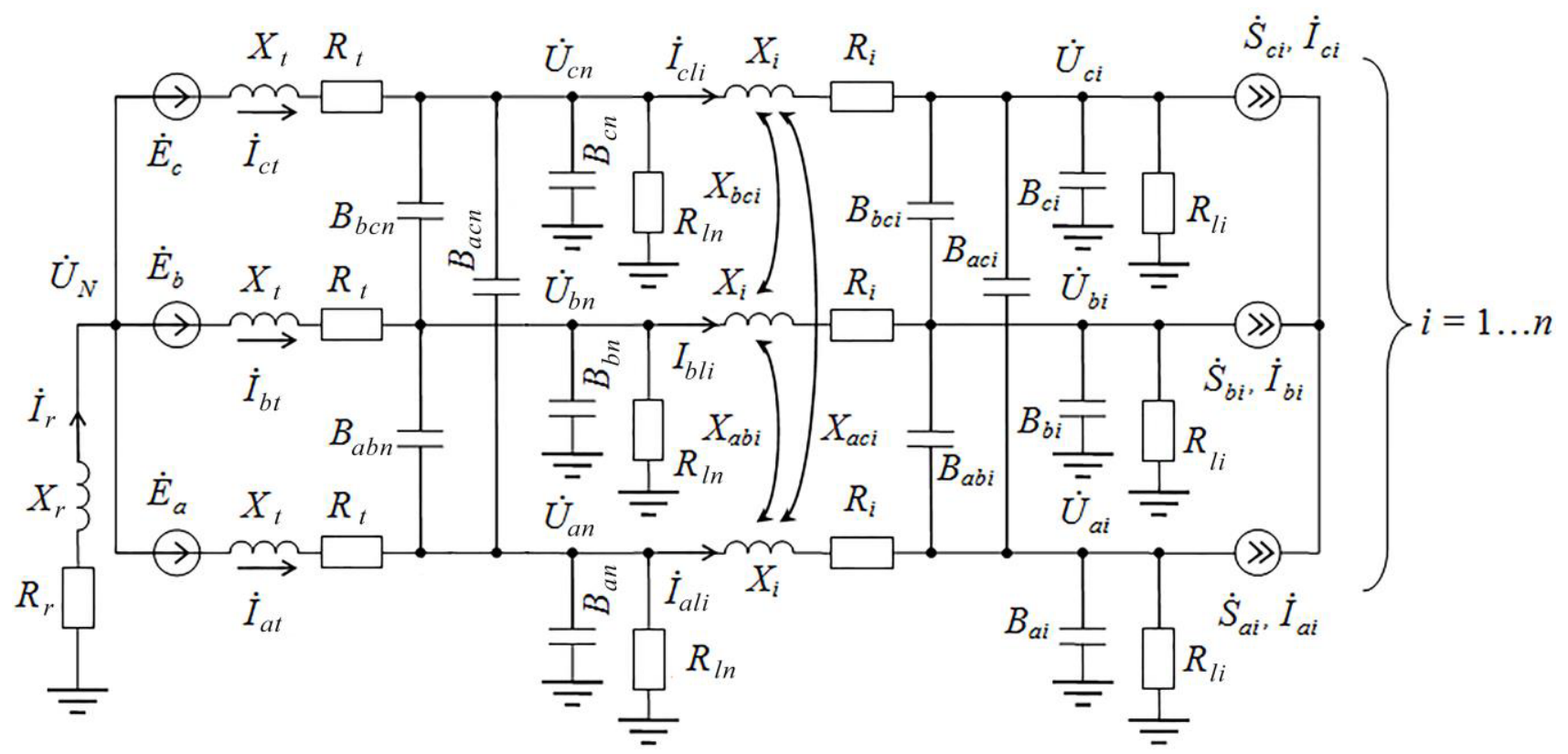

Figure 2. Equivalent circuit of $35 \mathrm{kV}$ radial network with arc-suppression coils

\section{Mathematical model}

Radial network of $35 \mathrm{kV}$ with arc-suppression coils are shown in Fig. 1 and its equivalent circuit are shown in Fig. 2.

The reason of the mode asymmetry is in nonequivalence of the geometric position of the overhead lines phase wiring. The result is in asymmetric own capacities, phase to phase (reciprocal) capacities [11] as well as the mutual inductance of each line.

The mathematic model is based on the method of phase coordinates. The allowances are:

1. The medium voltage winding of the transformer is considered as a three-phase source of EMF ( $\dot{E}_{a}=$ const, $\dot{E}_{b}=$ const, $\dot{E}_{c}=$ const ). The EMF can be either symmetrical or asymmetrical.

2. Load is given with constant power, which corresponds to the actual source data and considers nonlinear (in the inertial sense) character of consumers. When calculating the load currents one eliminates first voltage component of zero-sequence $\dot{U}_{a 12 i}, \dot{U}_{b 12 i}, \dot{U}_{c 12 i}$ and then current component of the zero sequence $\dot{I}_{0 i}, \dot{I}_{a i}$, $\dot{I}_{b i}, \dot{I}_{c i}$.

The system of mode equations on $a$ phase has the form by equation (1)-(27):

$$
\begin{gathered}
\dot{U}_{a 12 i}=\frac{1}{3}\left(2 \dot{U}_{a i}-\dot{U}_{b i}-\dot{U}_{c i}\right) \\
\dot{U}_{a n}=\dot{U}_{N}+\dot{E}_{a}-\dot{I}_{a t}\left(R_{t}+j X_{t}\right) \\
\dot{I}_{0 i}=\frac{1}{3}\left(\frac{S_{a i}^{*}}{U_{a 12 i}^{*}}+\frac{S_{b i}^{*}}{U_{b 12 i}^{*}}+\frac{S_{c i}^{*}}{U_{c 12 i}^{*}}\right)
\end{gathered}
$$

$$
\begin{gathered}
\dot{I}_{a i}=\frac{S_{a i}^{*}}{U_{a 12 i}^{*}}-\dot{I}_{0 i} \\
\dot{I}_{a l i}=\dot{I}_{a i}+\dot{U}_{a i}\left[\frac{1}{R_{l i}}+j\left(B_{a i}+B_{a b i}+B_{a c i}\right)\right] \\
-j \dot{U}_{b i} B_{a b i}-j \dot{U}_{c i} B_{a c i} \\
\dot{I}_{a t}=\sum_{i=1}^{n} \dot{I}_{a l i}+\dot{U}_{a n}\left[\frac{1}{R_{l n}}+j\left(B_{a n}+B_{a b n}+B_{a c n}\right)\right] \\
-j \dot{U}_{b n} B_{a b n}-j \dot{U}_{c n} B_{a c n} \\
\dot{I}_{r}=\dot{I}_{a t}+\dot{I}_{b t}+\dot{I}_{c t} \\
\dot{U}_{N}=-\dot{I}_{r}\left(R_{r}+j X_{r}\right) \\
\dot{U}_{a i}=\dot{U}_{a n}-\dot{I}_{a l i}\left(R_{i}+j X_{i}\right)-j \dot{I}_{b l i} X_{a b i}-j \dot{I}_{c l i} X_{a c i} \\
C_{0, a b}=-\beta_{a b}, \quad C_{0, a c}=-\beta_{a c} \\
B_{a b n}=0.5 \omega \sum_{i=1}^{n} C_{0, a b, i} l_{i} \\
B_{a b i}=0.5 \omega C_{0, a b, i} l_{i} \\
\end{gathered}
$$




$$
\begin{aligned}
& \boldsymbol{\beta}=\left(\begin{array}{ccc}
\beta_{a} & \beta_{a b} & \beta_{a c} \\
\beta_{b a} & \beta_{b} & \beta_{b c} \\
\beta_{c a} & \beta_{c b} & \beta_{c}
\end{array}\right)=\boldsymbol{\alpha}^{-\mathbf{1}} \\
& \boldsymbol{\alpha}=\left(\begin{array}{ccc}
\alpha_{a} & \alpha_{a b} & \alpha_{a c} \\
\alpha_{b a} & \alpha_{b} & \alpha_{b c} \\
\alpha_{c a} & \alpha_{c b} & \alpha_{c}
\end{array}\right) \\
& \alpha_{i, i}=\frac{1}{2 \pi \varepsilon_{0}} \ln \frac{2 h_{i}}{r_{i}} \\
& \alpha_{i, j}=\alpha_{j, i}=\frac{1}{2 \pi \varepsilon_{0}} \ln \frac{r_{i, j}^{\prime}}{r_{i, j}} \\
& r_{i, j}^{\prime}=\sqrt{r_{i, j}^{2}+4 h_{i} h_{j}} \\
& X_{i}=\omega\left(\frac{\mu_{0} l_{i}}{2 \pi}\left(\ln \frac{2 l_{i}}{r_{i}}-1\right)+\frac{\mu l_{i}}{8 \pi}\right) \\
& X_{a b i}=\omega \frac{\mu_{0} l_{i}}{2 \pi}\left(\ln \frac{2 l_{i}}{r_{a b, i}}-1\right) \\
& X_{b c i}=2 \cdot 10^{-7} \omega l_{i}\left(\ln \frac{2 l_{i}}{r_{b c, i}}-1\right) \\
& X_{a c i}=2 \cdot 10^{-7} \omega l_{i}\left(\ln \frac{2 l_{i}}{r_{a c, i}}-1\right) \\
& R_{l n}=\frac{2 U_{n o m}^{2}}{\sum_{i=1}^{n} \Delta P_{1,0, i} l_{i}}, R_{l i}=\frac{2 U_{n o m}^{2}}{\Delta P_{1,0, i} l_{i}} \\
& X_{r}=\frac{k_{e}}{\omega \sum_{i=1}^{n}\left(C_{0, a, i}+C_{0, b, i}+C_{0, c, i}\right) l_{i}} \\
& R_{r}=\Delta P_{r} \frac{U_{\mathrm{nom}, r}^{2}}{S_{\mathrm{nom}, r}^{2}}+R_{g}
\end{aligned}
$$

where $i=1 \ldots n ; n$ is a number of lines; $\dot{U}_{a 12 i}, \dot{U}_{b 12 i}$, $\dot{U}_{c 12 i}$ are voltage component of the zero sequence $\dot{U}_{a i}, \dot{U}_{b i}, \dot{U}_{c i}$ are phase voltages at the end of the line; $\dot{U}_{a n}, \dot{U}_{b n}, \dot{U}_{c n}$ are $a, b, c$ phase voltages respectively the neutral conductor; $\dot{U}_{N}$ is neutral displacement voltage; $\dot{E}_{a}$ is EMF of $a$ phase of the power supply which is regarded as a booster; $\dot{I}_{a t}, \dot{I}_{b t}, \dot{I}_{c t}$ are currents of $a, b, c$ phases flowing through the booster; $R_{t}, X_{t}$ are resistance and inductance of the low-voltage winding feeder transformer; $\dot{I}_{0 i}, \dot{I}_{a i}, \dot{I}_{b i}, \dot{I}_{c i}$ are current component of the zero sequence; $S_{a i}^{*}, S_{b i}^{*}, S_{c i}^{*}$ are load power; $\dot{I}_{a l i}, \dot{I}_{b l i}, \dot{I}_{c l i}$ are linear currents of $a, b, c$ phases; $R_{l n}, R_{l i}$ are resistances to surface-leakage currents through the insulators at the start and in the end respectively; $B_{a i}, B_{a b i}, B_{a c i}, B_{a b n}, B_{a c n}$, $B_{a n}$ are internal and mutual capacity admittance of the line; $\dot{I}_{r}$ is reactor current; $R_{r}, X_{r}$ are resistance and inductance of the reactor; $X_{i}$ is internal inductive resistance of the line phase; $X_{a b i}, X_{a c i}$ are mutual impedance of the line phases; $R_{i}$ is resistance in the line; $\omega$ is angular frequency; $C_{0, a, i}, C_{0, b, i}, C_{0, c, i}, C_{0, a b, i}, C_{0, a c, i}$ are linear phase and phase-tophase capacitances; ; $l_{i}$ is the length of the line; $\beta$ is a coefficient matrix of electrostatic induction; $\alpha$ is the matrix of potential coefficients; $\varepsilon_{0}=8.85 \cdot 10^{-12} \mathrm{~F} / \mathrm{m}$ is the dielectric constant; $h_{i}$ is the height of the phase wire on the ground; $r_{i}$ is the conductor radius; $r_{i, j}$ is the distance between the phase wires $\mathrm{i}$ and $\mathrm{j} ; r_{i, j}^{\prime}$ is the distance from the wire $i$ to $j$ mirroring the wire relative to the surface of the earth; $\mu_{0}=4 \pi \cdot 10^{-7} \mathrm{H} / \mathrm{m}$ is a magnetic constant; $\mu$ is absolute magnetic permeability of the conductor material taken equal to $\mu_{0} ; S_{\text {nom, }, r}, U_{n o m, r}$ are rated power and rated voltage of the reactor; $\Delta P_{l, 0, i}$ is linear power loss in insulators; $R_{g}$ is the equivalent earth resistance between the lines and the reactor capacities; $k_{e}$ is a coefficient considering the inaccuracy of adjustment; $\Delta P_{r}$ is rated active power losses; $U_{\text {nom }}$ is rated supply voltage.

Equations (1), (3) and (4) simulated electrical loads with the exclusion of the zero sequence currents. Equation (2) expresses the relation of phase voltage at the beginning of the line and neutral bias on the basis of the Kirchhoff's second law, and equation (9) expresses voltage relationships at the beginning and at the end of the line considering the mutual inductance. Equation (8) defines a neutral voltage displacement as the voltage drop at the reactor resistances. Equations (4), (5) and (6) represent the first law of Kirchhoff for the nodes at the end of the line, at the beginning of the line and the connection point of the reactor respectively. Capacitive admittances of the lines are determined with equations (10-20) based on the matrix inversion of potential coefficients (17). Self and mutual inductances of the lines are calculated with equations (21-24) via the geometric parameters. The mathematical model considers active resistances of the transverse lines of the branches, which are calculated according to equation (25) through the losses per unit length in insulators. Equation (27) allows to consider ground resistance together with the active impedance of the reactor. Equation (26) represents an equation of the reactor setting to the phase capacities of the lines. The complete system of the mode equations is obtained with adding to equations (1-27) of a phase equations describing the phase $\mathrm{b}$ and phase $\mathrm{c}$, which are recorded similarly.

\section{Simulation and results}

The results of the numerical solution of the complete system of equations for the radial $35 \mathrm{kV}$ network with 
three lines are presented. Initial data are given in Table 1. The MV winding of the transformer is considered as the source of three-phase EMF with internal resistance. Geometric parameters are defined for the lines, as well as active resistance per unit length and losses per unit length in insulators. Consumers capacities included at the end of each line are assumed to data on the lines. The reactor is set only to the resistance, because the inductive reactance is determined with the setting equation.

Table 1. Initial data for calculations

\begin{tabular}{|c|c|c|c|}
\hline Element & Parameter & Value & Note \\
\hline \multirow{3}{*}{$\begin{array}{l}\text { The booster TDTN- } \\
\text { 25000/110 (MV winding) }\end{array}$} & Resistance $R_{t}$ & $0.166 \mathrm{Ohms}$ & \multirow{2}{*}{ Given to medium voltage } \\
\hline & Inductive impedance $X_{t}$ & 0 & \\
\hline & Modules of phase EMF $E_{a}=E_{b}=E_{c}$ & $35 / \sqrt{3} \mathrm{KV}$ & Form symmetric system \\
\hline \multirow{6}{*}{$\begin{array}{l}\text { Lines (general } \\
\text { information) }\end{array}$} & $\begin{array}{l}\text { The height of the phase wires A and } \\
\qquad C h_{a}=h_{c}\end{array}$ & $15 \mathrm{~m}$ & \multirow{5}{*}{$\begin{array}{c}\text { Typical intermediate } \\
\text { steel single circuit tower } \\
{[12]}\end{array}$} \\
\hline & The height of the phase wire $\mathrm{B} h_{b}$ & $18 \mathrm{~m}$ & \\
\hline & Phase spacing $r_{a b}$ & $5 \mathrm{~m}$ & \\
\hline & Phase spacing $r_{b c}$ & $3.3 \mathrm{~m}$ & \\
\hline & Phase spacing $r_{a c}$ & $5.3 \mathrm{~m}$ & \\
\hline & Linear power loss in insulators $\Delta P_{l, 0}$ & $0.3 \mathrm{kWh} / \mathrm{km}$ & $\begin{array}{c}\text { On the basis of } \\
\text { generalized data [13] }\end{array}$ \\
\hline \multirow{4}{*}{$\begin{array}{l}\text { Line 1, } \\
\text { AS-120/19 }\end{array}$} & Length & 8 kilometers & \\
\hline & The radius of the wire & $7.6 \mathrm{~mm}$ & \\
\hline & Linear resistance $r_{0}$, & $0.2492 \mathrm{ohms} / \mathrm{km}$ & \\
\hline & Load power $\dot{S}$ & $6,6+j 3 \mathrm{MBA}$ & $\begin{array}{c}\text { Uniform distribution on } \\
\text { phases }\end{array}$ \\
\hline \multirow{4}{*}{$\begin{array}{c}\text { Line } 2 \\
\text { AS-95/16 }\end{array}$} & Length & 8 kilometers & \\
\hline & The radius of the wire & $6.75 \mathrm{~mm}$ & \\
\hline & Linear resistance $r_{0}$, & $0.306 \mathrm{Ohm} / \mathrm{km}$ & \\
\hline & Load power $\dot{S}$ & $4.5+j 3.6 \mathrm{MBA}$ & $\begin{array}{l}\text { Uniform distribution on } \\
\text { phases }\end{array}$ \\
\hline \multirow{4}{*}{$\begin{array}{l}\text { Line } 3 \text {, } \\
\text { AS } 70 / 11\end{array}$} & Length & $10 \mathrm{~km}$ & \\
\hline & The radius of the wire & $5.7 \mathrm{~mm}$ & \\
\hline & Linear resistance $r_{0}$, & $0.4286 \mathrm{ohms} / \mathrm{km}$ & \\
\hline & Load power $\dot{S}$ & $3.6+j 2.25 \mathrm{MBA}$ & $\begin{array}{l}\text { Uniform distribution on } \\
\text { phases }\end{array}$ \\
\hline Reactor & Resistance & $10.6 \mathrm{ohn}$ & \\
\hline
\end{tabular}

Table 2. The results of the set modes calculations

\begin{tabular}{|c|c|c|c|c|c|c|c|c|c|}
\hline \multicolumn{2}{|c|}{ Reactor adjustment $k_{e}$} & 0.8 & 0.84 & 0.88 & 0.9 & 0.92 & 0.94 & 0.96 & 0.98 \\
\hline \multirow{3}{*}{$\begin{array}{l}\text { Voltage in the } \\
\text { end of line } 1, \mathrm{kV}\end{array}$} & $U_{a 1}$ & 21.87 & 22.19 & 22.46 & 22.54 & 22.56 & 22.49 & 22.34 & 22.11 \\
\hline & $U_{b 1}$ & 19 & 19.2 & 19.61 & 19.92 & 20.28 & 20.68 & 21.1 & 21.5 \\
\hline & $U_{c 1}$ & 18.64 & 18.18 & 17.58 & 17.24 & 16.9 & 16.59 & 16.34 & 16.18 \\
\hline \multirow{3}{*}{$\begin{array}{l}\text { Currents in the } \\
\text { transformer, A }\end{array}$} & $I_{a t}$ & 288.8 & 288.8 & 288.9 & 288.9 & 288.9 & 288.9 & 288.9 & 288.9 \\
\hline & $I_{b t}$ & 288.7 & 288.7 & 288.7 & 288.7 & 288.7 & 288.7 & 288.7 & 288.7 \\
\hline & $I_{c t}$ & 289 & 289 & 289 & 289 & 289 & 289 & 289 & 289 \\
\hline \multicolumn{2}{|c|}{$\begin{array}{c}\text { Neutral displacement voltage } \\
U_{N}, \mathrm{~kW} \\
\end{array}$} & 2.106 & 2.484 & 2.895 & 3.097 & 3.285 & 3.446 & 3.568 & 3.644 \\
\hline \multicolumn{2}{|c|}{ The current in the reactor $\mathrm{I}_{r}, \mathrm{~A}$} & 0.288 & 0.324 & 0.36 & 0.377 & 0.391 & 0.402 & 0.407 & 0.407 \\
\hline \multicolumn{2}{|c|}{$\begin{array}{c}\text { Total active power losses } \\
\qquad \Delta P, \mathrm{~kW}\end{array}$} & 273.4 & 273.5 & 273.5 & 273.5 & 273.6 & 273.6 & 273.6 & 273.6 \\
\hline
\end{tabular}

Calculations were made for the overcompensation modes, which correspond to the value $k_{e}<1$ in (26). This choice is due to the fact that the undercompensation mode and the more accurate adjustment can lead to resonance neutral displacement voltage and therefore are not recommended or even are unacceptable. The main results of the calculations are presented in Table 2. These results include: 1) the voltage at the load nodes (at the end of the lines), which determines the quality of electricity; 2) neutral voltage displacement, which determines the tolerance of this setting; 3) current in a reactor as an additional parameter which characterizes the degree of 
asymmetry of the mode; 4) the total active power losses in the network. It is evident that the load voltage is substantially non-symmetrical.

However, it has little effect on the quality of electricity, because the asymmetry is mainly due to zero sequence voltage, which is filtered when passing through the stepdown transformers. Power losses in the network when approaching the resonance setting of the reactor increase, but a little. They are essentially the same in all modes as in the absence of the reactor $(273.4 \mathrm{~kW})$. It is due to the small asymmetry of the currents in the network and it indicates the energy efficiency of the considered modes.

\section{Conclusions}

1. In the table 2 is presented the voltages at the end of line 1 (the results for the other lines are similar). Real values of these voltages can be obtained only on the basis of models appropriate to the accuracy considered. Simplified models, not considering the load, will lead either to overrated voltage value at the end of the lines (if

you consider the longitudinal branch), or to the voltage equation at the beginning and the end of lines (with neglect of the longitudinal branches).

2 . If the voltage is noticeably asymmetrical, then for currents asymmetry is much less expressed (Table 2 shows the currents in the transformer, similar results are obtained for currents in the lines.) It should be expected, because the load is not sensitive to the residual voltage which is observed in practice. It proves the adequacy of the adopted model of the load.

3. Neutral displacement voltage increases monotonically with increasing reactor inductivity. The mode is expected as it is close to resonance. However, instead of the expected growth of voltage rise one observes some slowing. Thus, the neutral displacement voltage is more stable than it is obtained with the simplified model. Under these conditions, it is estimated around $10 \%$ from the nominal line voltage.

4. The presence of arcing reactor and changing its settings almost do not increase the energy losses in the network. Thus, the considered mode of the neutral operation is energy efficient.

The mathematical model can be used for a comprehensive analysis of steady-state modes of radial 35 $\mathrm{kV}$ networks with arc reactor, including the validation settings of the reactor and assessment of voltage quality.
In addition, this model provides a realistic initial conditions for calculating the overvoltage in the singlephase earth faults.

\section{Acknowledgment}

The paper was prepared in Omsk State Technical University with financial support from Ministry of Education and Science in the framework of the agreement No. 14.577.21.0097 on August 22, 2014. A unique identifier of applied research RFMEFI57714X 0097.

\section{References}

1. X. T. Wang, J. M. Lin, L. G. Ban, Z. T. Xiang, and B. Han, Power Syst. Techn., 33, 5, pp. 1-5, (2009)

2. K. Tao, and M.B.Liu, Power Syst. Protec. and Contr., 38, 16, pp. 39-43, (2010)

3. S. Zhong and A. Abur, in Proc. IEEE Power Eng. Soc.Winter Meeting, pp. 975-979, (2002)

4. L. Zou, F. Lin, Z. Long, J. Xie, Power Syst. Techn., 32, 2, pp. 283-286, (2008)

5. R. Burgess, and A. Ahfock, Australian Journ. of Electr. \& Electron. Eng., 10, 1, pp. 127-136, (2013)

6. M. Qian, B. Zheng ; L. Ban, and Z. Xiang, IEEE Trans. Power Del., 30 , 3, pp. 1458 - 1466, (2015).

7. Z. Xu, X. Yan, X. Zhang, and A. Wen, IET Generat., Transmiss., Distrib., 7, 1, pp. 1-8, (2013).

8. Zadeh M.R.D., Sanaye-Pasand M., Kadivar A., IEEE Trans. Power Del., 23, pp. 2472-2479, (2008).

9. V. Leitloff, L. Pierrat, and R. Feuillet, Eur. Trans. Elect. Power Eng, 4, pp. 145-153, (1994)

10. I.M. Sirota, S.N. Kislenko, and A.M. Mikhailov, The neutral electrical networks, Kiev: Nauk.Dumka, 264 p., (1985).

11. K.S.Demirchian, L.R.Neumann, N.V.Korovkin, and V.L.Chechurin, Theory of Electrical Engineering: In $3 \mathrm{~V}$, The textbook for high schools, 3, SPb: Peter, 377 p., (2006).

12. V.G.Gerasimov, Electrical reference: In 4 V. V.3. Production, transfer and distribution of electric energy, M .: MPEI Publishing, 964 p., (2002).

13. Yu.S. Zhelezko, A.V Artemyev, O.V. Savchenko Calculation, analysis and valuation of losses in electric networks: a guide for practical calculations, M .: Publishing House of NTs ENAS, 280 p., (2002). 